\title{
BioéthiqueOnline
}

\section{Continuité et discontinuité dans les trajectoires de soins de personnes ayant une problématique complexe de santé : perceptions d'ergothérapeutes - une étude exploratoire}

\section{Édith Rondeau-Boulanger et Marie-Josée Drolet}

Volume 5, 2016

URI : https://id.erudit.org/iderudit/1044266ar

DOI : https://doi.org/10.7202/1044266ar

Aller au sommaire du numéro

Éditeur(s)

BioéthiqueOnline

ISSN

1923-2799 (numérique)

Découvrir la revue

Citer cet article

Rondeau-Boulanger, É. \& Drolet, M.-J. (2016). Continuité et discontinuité dans les trajectoires de soins de personnes ayant une problématique complexe de santé : perceptions d'ergothérapeutes - une étude exploratoire.

BioéthiqueOnline, 5. https://doi.org/10.7202/1044266ar
Résumé de l'article

Depuis la mise sur pied d'un système de santé financé par des fonds publics et dirigé par un ministère de la santé, le réseau de la santé du Québec a connu plusieurs changements. Par exemple, des changements ont été réalisés au début des années 2000, visant en outre à améliorer l'accessibilité et la continuité des soins de santé disponibles à la population québécoise. En dépit du souci du Ministère d'améliorer la continuité des soins, des problèmes persistent pour les personnes présentant une problématique complexe de santé. En effet, les individus aux prises avec une comorbidité, c'est-à-dire avec un problème de santé physique et une autre de santé mentale, sont plus susceptibles de vivre des discontinuités dans leurs trajectoires de soins de santé (autrement dit une discontinuité dans leur accès aux soins), constituant une réponse peu adéquate à leurs besoins. Pour comprendre ce que vivent des ergothérapeutes qui desservent de tels clients, une étude exploratoire a été réalisée. Le but de cette recherche était d'explorer les perceptions d'ergothérapeutes à propos de la continuité et de la discontinuité dans les trajectoires de soins de ces clients. Les ergothérapeutes identifient des raisons qui, à leur avis, expliquent les discontinuités dans les trajectoires de soins des individus présentant une problématique complexe de santé, nomment plusieurs conséquences négatives de ces discontinuités et mentionnent des façons pouvant contribuer à améliorer la continuité des soins prodigués à ces personnes. La prise en compte des perceptions des ergothérapeutes s'avère pertinente afin d'améliorer les soins offerts à ces personnes.
Droits d'auteur (C É Rondeau-Boulanger et M-J Drolet, 2016

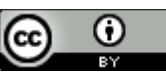

Ce document est protégé par la loi sur le droit d'auteur. L’utilisation des services d’Érudit (y compris la reproduction) est assujettie à sa politique d'utilisation que vous pouvez consulter en ligne. 


\title{
Continuité et discontinuité dans les trajectoires de soins de personnes ayant une problématique complexe de santé : Perceptions d'ergothérapeutes - une étude exploratoire
}

\author{
ARTICLE (RÉVISION PAR LES PAIRS / PEER-REVIEWED) \\ Édith Rondeau-Boulanger ${ }^{1}$, Marie-Josée Drolet ${ }^{2}$

Reçu/Received: 19 Mar $2015 \quad$ Publié/Published: 21 Apr 2016
Éditeurs/Editors: Charles Dupras, Vanessa Chenel, Marie-Eve Lemoine
Évaluateurs externes/Peer-Reviewers: Josée Lemoignan \& Louise Lafortune

2016 É Rondeau-Boulanger, M-J Drolet, Creative Commons Attribution 4.0 International License

\section{Résumé}

Depuis la mise sur pied d'un système de santé financé par des fonds publics et dirigé par un ministère de la santé, le réseau de la santé du Québec a connu plusieurs changements. Par exemple, des changements ont été réalisés au début des années 2000 , visant en outre à améliorer l'accessibilité et la continuité des soins de santé disponibles à la population québécoise. En dépit du souci du Ministère d'améliorer la continuité des soins, des problèmes persistent pour les personnes présentant une problématique complexe de santé. En effet, les individus aux prises avec une comorbidité, c'est-à-dire avec un problème de santé physique et une autre de santé mentale, sont plus susceptibles de vivre des discontinuités dans leurs trajectoires de soins de santé (autrement dit une discontinuité dans leur accès aux soins), constituant une réponse peu adéquate à leurs besoins. Pour comprendre ce que vivent des ergothérapeutes qui desservent de tels clients, une étude exploratoire a été réalisée. Le but de cette recherche était d'explorer les perceptions d'ergothérapeutes à propos de la continuité et de la discontinuité dans les trajectoires de soins de ces clients. Les ergothérapeutes identifient des raisons qui, à leur avis, expliquent les discontinuités dans les trajectoires de soins des individus présentant une problématique complexe de santé, nomment plusieurs conséquences négatives de ces discontinuités et mentionnent des façons pouvant contribuer à améliorer la continuité des soins prodigués à ces personnes. La prise en compte des perceptions des ergothérapeutes s'avère pertinente afin d'améliorer les soins offerts à ces personnes.

\section{Mots clés}

continuité des soins, discontinuités, trajectoires de soins, problématique complexe de santé, comorbidité, ergothérapie, holisme, phénoménologie

\section{Abstract}

Since the creation of a health system financed by public funds and managed by the Ministry of Health, the Quebec health network has undergone numerous changes. For example, changes were made in early 2000 in order to improve the accessibility and continuity of healthcare available to Quebecers. Despite the concern of the Ministry to improve continuity of care, problems persist for people with complex health problems. Indeed, individuals struggling with comorbidity - e.g., with both physical and mental health problems - are more likely to experience discontinuities in their health care trajectories (i.e., discontinuity in their access to care), constituting an inadequate response to their needs. To understand the experiences of occupational therapists that serve such clients, an exploratory study was conducted. The purpose of this research was to explore perceptions of occupational therapists about continuity and discontinuity in the care trajectories of these clients. Occupational therapists identified the reasons which, in their opinion, explained the discontinuities in the trajectory of care individuals with complex health problems, and some of the negative consequences of these discontinuities; they also noted ways to improve the continuity of care for these clients. Taking into account the perceptions of occupational therapists is important in order to improve care for people with complex health problems.

\section{Keywords}

continuity of care, discontinuities, care trajectories, complex health problems, comorbidity, occupational therapy, holism, phenomenology

\section{Responsabilités des évaluateurs externes}

Les évaluations des examinateurs externes sont prises en considération de façon sérieuse par les éditeurs et les auteurs dans la préparation des manuscrits pour publication. Toutefois, être nommé comme examinateur n'indique pas nécessairement l'approbation de ce manuscrit. Les éditeurs de BioéthiqueOnline assument la responsabilité entière de l'acceptation finale et la publication d'un article.

\section{Peer-reviewer responsibilities}

Reviewer evaluations are given serious consideration by the editors and authors in the preparation of manuscripts for publication. Nonetheless, being named as a reviewer does not necessarily denote approval of a manuscript; the editors of BioéthiqueOnline take full responsibility for final acceptance and publication of an article. 
Affiliations des auteurs / Author Affiliations

${ }^{1}$ Centre intégré universitaire de santé et de services sociaux de la Mauricie et du Centre-du-Québec (CIUSSS-MCQ), Trois-Rivières, Canada

${ }^{2}$ Département d'ergothérapie, Université du Québec à Trois-Rivières (UQTR), Trois-Rivières, Canada

\section{Correspondance / Correspondence}

Édith Rondeau-Boulanger, edith.rondeauboulanger@gmail.com

\section{Remerciements}

Les auteures remercient chaleureusement les participants à cette recherche. Cette étude a pu être réalisée grâce à eux et a permis aux auteures d'explorer un sujet qui les intéressait grandement. Elles remercient également les étudiants de la cohorte d'Ergothérapie 2010-2014 de I'UQTR, qui demeurent une source d'inspiration à maints égards.

\section{Conflit d'intérêts}

Aucun déclaré

\section{Acknowledgements}

The authors gratefully acknowledge the participants in this study. This study was made possible thanks to them and allowed the authors to explore a topic of great interest. The authors also thank the students of the 2010-2014 UQTR Occupational Therapy cohort, who remain an inspiration in many ways.

\section{Conflicts of Interest}

None to declare

\section{Introduction ${ }^{1}$}

Le réseau de la santé et des services sociaux (RSSS) du Québec, tel que nous le connaissons aujourd'hui, est relativement jeune. Fondé à l'origine par les communautés religieuses, il est sous la gouverne de l'État québécois depuis une cinquantaine d'années seulement $[1,2]$. Depuis la mise sur pied d'un système de santé financé par des fonds publics et dirigé par le Ministère de la Santé et des Services sociaux (MSSS), le RSSS du Québec a connu plusieurs changements importants. Conséquemment, ceux qui sont en cours ne font suite qu'à plusieurs autres. Par exemple, des changements ont été réalisés au début des années 2000 visant à améliorer l'accessibilité et la continuité des soins de santé ${ }^{2}$ disponibles à la population québécoise, notamment aux personnes présentant une problématique complexe de santé, soit des personnes présentant en concomitance ${ }^{3}$ au moins un problème de santé mentale et au moins un problème de santé physique [4]. II s'agissait alors d'assurer non seulement l'intégration verticale, mais également et surtout l'intégration horizontale des soins considérées à l'époque comme déficientes [5]. Précisons que l'intégration verticale des soins vise à offrir aux individus un accompagnement de santé de leur naissance à leur mort, ce qui inclut la prévention et les soins de fins de vie. Pour ce faire, les mécanismes de référence et les processus de hiérarchisation des soins doivent être bien établis, et ce, dans le but de minimiser les listes d'attente et de mettre sur pied des trajectoires de soins continues et efficaces. L'intégration horizontale cherche, quant à elle, à offrir une réponse optimale à la multiplicité des besoins de santé d'une personne. Pour ce faire, une offre de services complémentaires, des partenariats et des collaborations efficaces sont nécessaires, et ce, dans le but d'améliorer la globalité des soins, en particulier pour les personnes présentant une problématique complexe de santé. Ces deux types d'intégrations ${ }^{4}$ visent en fin de compte à éviter la duplication des soins, les incohérences et les discontinuités ${ }^{5}$ dans les trajectoires de soins de santé des personnes [7]. Or, en dépit de cette volonté

\footnotetext{
${ }^{1}$ Le genre masculin est utilisé afin d'alléger le texte, sans aucune discrimination.

${ }^{2}$ Dans cet article, la notion de " soins » et l'expression " soins de santé » incluent non seulement les soins de santé, mais également les services sociaux, afin de simplifier le texte.

${ }^{3}$ Comme le constatent Buck et ses collaborateurs, la notion de comorbidité ou de concomitance est utilisée de façon hétérogène dans les écrits [3]. Dans cet article, la notion réfère au fait pour une personne d'avoir au moins un problème de santé physique combiné à au moins un problème de santé mentale. Ce faisant, cette personne présente une problématique complexe de santé.

${ }^{4}$ Mentionnons que, dans les écrits, d'autres définitions de l'intégration des soins sont utilisées [6].

${ }^{5}$ Par souci de simplification du propos, la notion de "discontinuité » dans les trajectoires de soins est opposée à la continuité dans les trajectoires de soins. Aussi, elle est synonyme de la notion de rupture dans les trajectoires de soins et peut aussi référer aux vides de soins ou de services.
} 
d'améliorer l'accessibilité et la continuité par une meilleure intégration verticale et horizontale des soins, des problèmes persistent, en particulier pour les personnes présentant une problématique complexe de santé, et ce, notamment eu égard au continuum des soins [5].

De fait, les personnes présentant une problématique de comorbidité, plus que d'autres clientèles, sont susceptibles de vivre des épisodes de discontinuités dans leurs trajectoires de soins de santé [5]. Voici deux exemples de ces discontinuités, voire de ces vides de services. 1) Les personnes qui ont un problème de santé mentale (ex. : une schizophrénie ou un trouble de personnalité) et dont le problème de santé physique est relativement mineur peuvent rencontrer des difficultés à avoir accès à un programme de réinsertion au travail. 2) Les individus qui ont un problème de toxicomanie et dont leur autre problème de santé est relativement mineur pourront avoir de la difficulté à accéder à des soins puisqu'on exigera parfois d'eux que leur problème de dépendance soit d'abord réglé. Paradoxalement, le personnel des centres de désintoxication aura une requête similaire pour leur autre problème de santé, confrontant ces personnes à une impasse : une impossibilité pour elles à recevoir des soins. Autrement dit, il est possible que les besoins relatifs à la santé physique ou ceux ayant trait à la santé mentale de ces personnes ne soient pas répondus ou qu'ils soient traités de façon distincte par des équipes différentes appartenant à des établissements séparés, et que ces soins ne soient pas coordonnés, occasionnant ainsi des discontinuités dans les trajectoires de soins de ces clients ou même des vides de services. Le même constat est fait dans d'autres pays, notamment en Angleterre [8,9] et en Australie [10-12], pour ne nommer que ces pays.

Mentionnons que selon l'Organisation mondiale de la Santé (OMS), une personne sur quatre est appelée à vivre un problème de santé mentale au cours de son existence [13]. Toujours selon l'OMS, $80 \%$ de ces personnes ne reçoivent pas les soins dont elles ont pourtant besoin [14]. Parmi ces individus, certains ont également un problème de santé physique, comme une lésion musculosquelettique, une blessure médullaire ou une amputation. Comme indiqué plus tôt, ces individus sont considérés, par le ministère de la Santé et des Services sociaux du Québec, comme des personnes présentant une problématique complexe de santé [5]. Soulignons que la principale expectative de ces individus, lorsqu'ils reçoivent des soins de santé, est d'avoir accès à des soins de qualité et en continu pendant leur expérience de soins, ce qui n'est pas entièrement le cas actuellement au Québec ${ }^{6}[5]$.

Bjorklund, Svensson et Read [16] se sont aussi intéressés aux discontinuités dans les trajectoires de soins de santé. Selon ces chercheurs, la valeur qu'est l'holisme pourrait être une solution à ce problème sociétale, car celle-ci pourrait faciliter une réponse adéquate aux besoins variés de ces personnes. Pour un ergothérapeute qui a une vision holistique et unifiée de l'être humain [7,16-24], le cloisonnement qui se présente de nos jours entre les établissements de santé dédiés à traiter les problèmes de santé mentale et ceux voués à traiter les problèmes de santé physique étonne, voire dérange. Pourquoi cette séparation entre les soins relatifs à la santé mentale des individus et ceux ayant trait à leur santé physique? Cette organisation ne contribue-t-elle pas à expliquer, à tout le moins en partie, les discontinuités dans les trajectoires de soins ou même les vides de services que vivent les individus ayant une problématique complexe de santé? En fait, pour un ergothérapeute qui a une vision holistique et unifiée de la personne, cette organisation dualiste des soins apparait, dans une certaine mesure, tel un legs du passé, en ceci qu'elle semble tributaire d'une vision cartésienne de l'être humain, selon laquelle l'âme serait en quelque sorte séparée et distincte du corps. Rappelons que chez Descartes, la substance pensante qu'est l'âme est distincte et séparée de la substance étendue qu'est le corps [25]. Pour le dire autrement, l'ergothérapeute qui valorise une anthropologie holistique, qui conçoit l'être humain comme un tout unifié, aura tendance à considérer comme obsolète toute vision dualiste de la personne humaine et tout cloisonnement des soins, et ce, surtout si cette vision et cette organisation semblent concourir à occasionner des discontinuités dans les trajectoires de soins de certaines personnes et, conséquemment, des réponses peu adéquates à

${ }^{6}$ Soulignons que ce phénomène s'observe également pour les Québécois ayant un trouble de santé mentale associé à un problème de toxicomanie [15]. 
leurs besoins. Car, pour l'ergothérapeute, l'être humain doit être appréhendé de façon globale, c'està-dire que toutes les dimensions de la personne (affective, cognitive, physique et sociale) doivent être considérées indissociablement puisqu'elles sont fondamentalement interreliées et interdépendantes : elles forment un tout unifié et indissociable [7,16-24]. C'est pourquoi l'ergothérapeute aura tendance à aborder de front l'ensemble de ces dimensions de la personne dans le cadre de sa pratique professionnelle, plutôt que de les aborder en silo [18]. La valeur qu'est l'holisme se présente, en ce sens, comme une valeur constitutive et importante de l'identité professionnelle des ergothérapeutes [17] et le cloisonnement actuel des soins est à maints égards en rupture avec cette vision globale et unifiée de la personne.

Le contexte actuel de la Loi 10 réitère le besoin de réfléchir aux manières d'améliorer la prestation actuelle des soins de santé au Québec, notamment pour les individus ayant une problématique complexe de santé dont la vulnérabilité s'avère souvent plus grande que d'autres clientèles et dont la réponse optimale à l'entièreté des besoins se voit de nos jours compromise [5]. Le cloisonnement entre les soins de santé physique et ceux de santé mentale au Québec peut-il expliquer, à tout le moins en partie, le manque d'intégration horizontale des soins? L'optimisation des soins ${ }^{7}$ incite-t-elle les intervenants à " contourner » les problèmes singuliers et multiples de ces personnes et à ne pas accueillir leur vécu propre au moment où celles-ci sont en quête de soins? Le contexte actuel rappelle aussi, nous semble-t-il, le besoin de réfléchir de manière critique à l'organisation et à la prestation des soins de santé, de façon à ne pas négliger de considérer les préoccupations et les bonnes idées des intervenants, en l'occurrence des ergothérapeutes, qui quotidiennement prodiguent des soins à des clients ayant de multiples besoins.

Bien que plusieurs professionnels de la santé aient une vision holiste de la personne, la valeur qu'est l'holisme constitue une part importante de l'essence de l'ergothérapie [17,19], d'où le choix d'ergothérapeutes pour conduire cette étude. Aussi, puisque cette valeur, si importante pour l'ergothérapeute, se voit compromise par l'organisation actuelle des soins, le risque que ces professionnels de la santé soient confrontés à des situations éthiques, notamment à de la détresse éthique $^{8}$ [27], est bel et bien réel. En effet, lorsque des professionnels de la santé rencontrent des barrières à l'actualisation de valeurs importantes pour eux, ils peuvent être confrontés à de la détresse éthique [28,29] et même à de l'épuisement professionnel [30]. De plus, si quelques études ont documenté les perceptions de clients ayant une comorbidité des discontinuités dans les trajectoires de soins [8-12,15], aucune recherche n'a, à notre connaissance, recueilli les perceptions d'intervenants du réseau de la santé, incluant les ergothérapeutes, de ce phénomène.

Pour comprendre ce que perçoivent des ergothérapeutes qui desservent des personnes ayant une problématique complexe de santé, une étude exploratoire a donc été réalisée [7]. Le but de cette recherche était d'explorer les perceptions d'ergothérapeutes de la continuité et des discontinuités dans les trajectoires de soins de santé de telles personnes. Plus précisément, la question à l'origine de cette étude était la suivante: Quelles sont les perceptions d'ergothérapeutes du continuum de soins et, s'il y en a, des ruptures de soins que vivent des personnes ayant une problématique complexe de santé? Ainsi, l'objectif de cette recherche était d'explorer les perceptions d'ergothérapeutes de la continuité et des discontinuités de soins que rencontrent des individus ayant une concomitance entre un problème de santé physique et un problème de santé mentale. Le but de cet article est de décrire les perceptions des ergothérapeutes rencontrés dans le cadre de cette étude.

\footnotetext{
7 L'optimisation des soins correspond à un processus visant à déterminer parmi les interventions possibles celles qui donnent de meilleurs résultats compte tenu des contraintes. La visée de l'optimisation est notamment l'augmentation du rendement compte tenu des ressources allouées à la santé, l'efficience des équipes, des programmes, des établissements. ${ }^{8}$ Selon Swisher et ses collaborateurs [26], un professionnel de la santé peut être confronté à différentes situations éthiques, notamment à de la détresse éthique. La détresse éthique se présente lorsqu'un professionnel rencontre une barrière à l'actualisation d'une valeur éthique légitime et désirable. Ce faisant, il peut vivre une certaine détresse psychologique.
} 


\section{Méthodes}

Le devis de recherche retenu pour répondre à la question à l'origine de cette étude exploratoire est un devis qualitatif inspiré de la phénoménologie [31]. Cette méthode est appropriée étant donné que cette recherche documente les perceptions d'ergothérapeutes de la continuité et des discontinuités dans les trajectoires de soins de santé que vivent des personnes ayant une problématique complexe de santé. Comme l'indiquent Hunt et Carnevale [32], le choix d'un devis qualitatif de type phénoménologique est approprié pour explorer l'expérience subjective de personnes confrontées à des situations préoccupantes d'un point de vue éthique, notamment parce que la phénoménologie vise à " comprendre un phénomène et à en saisir l'essence du point de vue des personnes qui en font ou en ont fait l'expérience » [33, p.35].

\section{Participants à la recherche}

Des ergothérapeutes ont été rencontrés afin de répondre à la question à l'origine de l'étude et d'atteindre l'objectif identifié. En vue de sélectionner les participants à l'étude, des critères d'inclusion et d'exclusion ont guidé leur recrutement. Les ergothérapeutes désirant participer à l'étude devaient : 1) être ergothérapeute diplômé et faire partie de l'Ordre des ergothérapeutes du Québec; 2) travailler auprès d'adultes ou de personnes âgées dans le domaine de la santé physique ou de la santé mentale dans le RSSS du Québec; et 3) s'exprimer en français et bien le comprendre. II était également souhaité que les ergothérapeutes aient une expérience variée en termes de milieux de pratique, d'années d'expérience et de clientèles. Bien entendu, les participants potentiels devaient souhaiter participer à l'étude et être disponibles au moment de la collecte des données, qui a eu lieu durant le mois de novembre 2014.

\section{Recrutement des participants}

Pour être en mesure de recueillir les perceptions des ergothérapeutes-participants, la méthode d'échantillonnage par choix raisonné a été privilégiée. Cette façon de procéder, suggérée par Fortin pour ce type d'étude [33], se décrit comme une méthode d'échantillonnage visant à choisir des personnes selon des critères définis, dans le but que les participants soient le plus représentatifs du phénomène à l'étude.

Les ergothérapeutes sélectionnés, en fonction des critères d'inclusion présentés ci-dessus, ont d'abord été contactés par téléphone par l'auteure principale (ÉRB) pour vérifier leur intérêt à participer à l'étude. Lorsque c'était le cas, un courriel leur a été transmis, lequel contenait une lettre d'information précisant tous les détails de la recherche et en quoi consistait exactement leur éventuelle participation à l'étude. Les ergothérapeutes devaient être enclins à accorder du temps à la recherche en dehors de leurs heures de travail. Les ergothérapeutes sollicités et recrutés dans le cadre de cette étude l'ont été puisqu'ils travaillaient soit dans des milieux différents ${ }^{9}$ ou auprès de clientèles ou programmes distincts. ${ }^{10}$ Enfin, étant donné que cette recherche s'inscrivait dans le cadre d'une maîtrise professionnelle en ergothérapie, la participation de cinq ergothérapeutes était visée.

\section{Outils de collecte des données}

L'entrevue semi-dirigée était tout indiquée pour la collecte des données qualitatives de l'étude (voir en annexe le schéma de l'entrevue). Comme l'observent Fontana et Frey, l'entrevue est le meilleur moyen pour tenter de saisir la réalité vécue par une personne [34]. Ainsi, aux fins de l'étude, un schéma d'entrevue a été développé, sur la base d'une recension des écrits. Le schéma de l'entrevue a été conçu de façon à ce que les questions soient regroupées par thèmes (par exemple : perceptions de la continuité et des discontinuités dans les trajectoires de soins des personnes ayant une problématique complexe de santé; perceptions des raisons et des conséquences de ces discontinuités s'il y en a; perceptions des enjeux éthiques soulevés par celles-ci et les pistes

\footnotetext{
${ }^{9}$ Par exemple : centre de réadaptation, Centre local de services communautaires (CLSC), milieux hospitaliers, etc.

${ }^{10}$ Par exemple : programme des traumatisés crâniaux-cérébraux, soutien à domicile, santé mentale, etc.
} 
d'amélioration possibles). Ceci était dans le but de dégager aisément les unités de sens découlant des différents thèmes abordés dans le cadre des entretiens. Le schéma de l'entrevue n'a pas été soumis à un processus de validation, étant donné la nature exploratoire de l'étude et le temps imparti pour conduire la recherche (quatre sessions consécutives). Bien que semi-dirigées, les questions étaient ouvertes afin de laisser toute la place aux perceptions des ergothérapeutes-participants. Les entretiens étaient d'environ 50 minutes, excluant l'achèvement du questionnaire sociodémographique qui a permis de recueillir des informations sur les participants. Les entrevues ont été enregistrées afin de faciliter leur transcription. Mentionnons que les participants ont été rencontrés entre le 20 octobre et le 25 novembre 2014, dans un lieu et à un moment de leur convenance.

\section{Analyse des données}

L'analyse des données a été réalisée suivant une méthode usuelle d'analyse de contenu, qui consiste à faire ressortir des catégories, des thèmes et des unités de sens saillants à partir des verbatim des entrevues semi-structurées qui ont été transcrites intégralement. Plus précisément, les cinq étapes de Giorgi qui permettent d'appliquer la méthode phénoménologique [35] ont été réalisées, soit : 1) la collecte des données qualitatives; 2) la lecture des données transcrites sous forme de verbatim; 3 ) la création des unités de sens compilés dans des tableaux d'extraction des données (les extraits des verbatim ont été regroupés selon leur similitude eu égard aux unités de sens dégagées); 4) l'organisation et la formulation des données dans le langage disciplinaire; et 5) la synthèse des résultats [36].

\section{Considérations éthiques}

Cette étude a été approuvée par le Comité d'éthique de la recherche avec des êtres humains (CÉREH) de l'Université du Québec à Trois-Rivières (UQTR). Un formulaire de consentement a été signé par chacun des participants. Mentionnons que les données ont été traitées dans la plus stricte confidentialité et que les participants étaient libres d'accepter, de refuser ou d'abandonner leur participation à l'étude à tout moment.

\section{Résultats}

Cette section présente les principaux résultats de l'étude, en débutant par une description des participants. Sont ensuite rapportées les perceptions des participants-ergothérapeutes concernant les soins prodigués aux personnes présentant une problématique complexe de santé et de leur continuité ou de leur discontinuité. Plus précisément, les résultats relatifs aux perceptions des ergothérapeutes sont présentés en fonction des trois thèmes suivants: les principales raisons qui expliquent les discontinuités dans les trajectoires des soins, les conséquences de ces ruptures et les pistes de solution proposées.

\section{Description de l'échantillon}

Cinq ergothérapeutes $(n=5)$ ont participé à cette recherche. Ces ergothérapeutes étaient toutes des femmes (par conséquent, le féminin sera dorénavant employé pour faire référence aux participants pour la suite du texte). Comme l'indique le Tableau 1, les cinq ergothérapeutes rencontrées dans le cadre de cette étude étaient, au moment de la collecte des données, âgées de 31 à 50 ans. La majorité des participantes-ergothérapeutes détenait un baccalauréat $(n=4)$. Les années d'expérience professionnelle variaient de 7 et 23 ans (moyenne : 17,5 ans). Comme souhaité, ces ergothérapeutes pratiquaient, au moment de la recherche, au sein de milieux de pratique différents, en santé physique ou santé mentale. Aussi, les participantes desservaient des clientèles adultes et des personnes âgées ayant des problèmes de santé variés, dont des clients présentant une problématique de comorbidité. 
Tableau 1 : Description des participantes-ergothérapeutes

\begin{tabular}{|l|c|c|c|c|c|}
\hline $\begin{array}{l}\text { Caractéristiques } \\
\text { des participantes }\end{array}$ & $\begin{array}{c}\text { Ergothérapeute } \\
\mathbf{n}^{\circ} \mathbf{1}\end{array}$ & $\begin{array}{c}\text { Ergothérapeute } \\
\mathbf{n}^{\circ} \mathbf{2}\end{array}$ & $\begin{array}{c}\text { Ergothérapeute } \\
\mathbf{n}^{\circ} \mathbf{3}\end{array}$ & $\begin{array}{c}\text { Ergothérapeute } \\
\mathbf{n}^{\circ} \mathbf{4}\end{array}$ & $\begin{array}{c}\text { Ergothérapeute } \\
\mathbf{n}^{\circ} \mathbf{5}\end{array}$ \\
\hline Âge & $41-50$ ans & $31-40$ ans & $41-50$ ans & $41-50$ ans & $31-40$ ans \\
\hline Scolarité & Baccalauréat & Baccalauréat & Baccalauréat & $\begin{array}{c}\text { Maîtrise } \\
\text { professionnelle }\end{array}$ & Baccalauréat \\
\hline $\begin{array}{l}\text { Années } \\
\text { d'expérience }\end{array}$ & 20 & 7 & 20 & 23 & 14 \\
\hline $\begin{array}{l}\text { Milieu de } \\
\text { pratique }\end{array}$ & $\begin{array}{c}\text { CRDP } \\
\text { (Santé physique) }\end{array}$ & $\begin{array}{c}\mathrm{CH} \\
(\text { Santé mentale) }\end{array}$ & $\begin{array}{c}\text { CRDP } \\
\text { (Santé physique) }\end{array}$ & $\begin{array}{c}\mathrm{CH} \\
\text { (Santé mentale) }\end{array}$ & $\begin{array}{c}\text { CLSC } \\
\text { (santé physique) }\end{array}$ \\
\hline
\end{tabular}

CRDP : Centre de réadaptation en déficience physique ; $\mathrm{CH}$ : Centre hospitalier ; CLSC : Centre local de services communautaires

\section{Raisons des discontinuités dans les trajectoires de soins}

Lorsqu'elles ont été questionnées au sujet des raisons des discontinuités dans les trajectoires de soins, toutes les participantes ont rapporté des discontinuités dans les trajectoires de soins des personnes présentant une problématique complexe de santé. Pour elles, il était évident que la continuité des soins constitue à l'heure actuelle un idéal plus qu'une réalité pour ces clients. Plus encore, toutes les ergothérapeutes-participantes ont proposé différentes raisons qui, à leur avis, contribuent à expliquer les discontinuités dans les trajectoires de soins que vivent les personnes présentant une problématique complexe de santé (Tableau 2).

Tableau 2 : Principales raisons des discontinuités dans les trajectoires de soins

\begin{tabular}{|l|c|}
\hline Raisons des discontinuités selon les participantes & $\begin{array}{c}\text { Nombre } \\
\text { d'ergothérapeutes }\end{array}$ \\
\hline Cloisonnement des soins de santé & 5 \\
\hline Suppressions de postes ou non-remplacements des congés & 5 \\
\hline Optimisation des soins de santé & 5 \\
\hline Complexité du problème de santé des clients & 5 \\
\hline Vides de services pour cette clientèle & 5 \\
\hline Désaccords entre les intervenants sur ce qui doit être fait & 4 \\
\hline Préjugés relatifs aux troubles de santé mentale & 4 \\
\hline Critères d'admission très stricts, voire discriminatoires & 3 \\
\hline Pression de rendement et de performance ressentie par les intervenants & 3 \\
\hline
\end{tabular}

Un des éléments qui fut rapporté par la totalité des participantes $(n=5)$ est que l'organisation actuelle du RSSS ne permet pas l'intégration horizontale des soins, notamment parce que celle-ci demeure cloisonnée à maints égards. Pour les ergothérapeutes, cette déficience sur le plan systémique se reflète par le manque d'opportunités actuellement disponibles aux intervenants de différents établissements de communiquer entre eux ou d'établir des plans de services entre les différents milieux de soins pour cette clientèle. Autrement dit, le cloisonnement des soins complique la collaboration entre les établissements et les intervenants, ce qui fait qu'il n'y a pas suffisamment d'informations transmises entre les différents milieux, rendant difficile la réponse adéquate à la multiplicité des besoins de ces personnes.

Un autre élément, discuté par toutes les participantes $(n=5)$, qui contribue, selon elles, à expliquer le manque d'intégration horizontale des soins a trait aux suppressions de postes de personnels et aux non-remplacements d'intervenants lors de congés (maladie, congé parental, etc.). Les postes coupés ou non comblés exacerbent, selon les participantes, la difficulté du RSSS à offrir des soins optimaux et continus aux personnes ayant une problématique complexe de santé et carrément, dans certains cas, à répondre à leurs besoins. Par exemple, l'ergothérapeute $n^{\circ} 3$ explique que lorsqu'on ne remplace pas le psychologue de l'équipe, les services psychologiques ne sont tout simplement pas offerts. Dans ce cas, étant donné leurs compétences professionnelles relatives à l'habilitation aux occupations et de leur champ de pratique, les ergothérapeutes tentent du mieux qu'ils peuvent 
d'assurer un certain confort psychologique, mais ils ne peuvent pas offrir des services psychologiques comme tels. Bien que les ergothérapeutes soient formés à la relation d'aide et qu'ils offrent un soutien psychologique à la clientèle, ils ne sont pas psychologues. Ces coupures et non-remplacements les affectent donc indirectement, mais les personnes les plus touchées demeurent en premier lieu les clients.

Ensuite, les cinq ergothérapeutes rencontrées ont toutes exprimé que la complexité des problèmes de santé de certains clients peut nuire à leur cheminement dans le continuum de soins, en outre parce que ceux-ci parviennent difficilement à défendre par eux-mêmes leurs droits. Effectivement, la complexité des problèmes de santé de certains de ces clients ne se réduit pas à une concomitance entre un problème de santé physique et un problème de santé mentale. II est possible que des personnes ayant des troubles cognitifs, un problème de toxicomanie, une déficience intellectuelle ou un début de démence vivent aussi ce genre de situations, particulièrement lorsque s'ajoute un problème de santé physique. L'ergothérapeute $\mathrm{n}^{\circ} 3$, à ce sujet, raconte ceci : "De toute façon, maintenant, c'est la complexité. Alors la concomitance... Cela peut être n'importe quoi. Quelqu'un qui a une DI [déficience intellectuelle], un problème de santé mentale et un problème de santé physique. Comment veux-tu qu'il défende ses droits? " Cet extrait reflète que la complexité d'un problème de santé vécue par une personne peut se manifester sous diverses formes et que l'ergothérapeute est un professionnel qui est appelé à travailler auprès de clients semblables, de même qu'à les représenter afin que leurs droits soient respectés.

Plusieurs participants rapportent qu'il existe non seulement des discontinuités dans les trajectoires de soins de ces personnes, mais également des vides de services pour cette clientèle $(n=5)$. Selon les participantes rencontrées, les vides de services peuvent survenir dans certains cas en raison de l'absence de tiers en mesure de financer les soins, comme la Commission de la santé et de la sécurité du travail (CSST ; maintenant la Commission des normes, de l'équité, de la santé et de la sécurité du travail, CNESST) ou la Société de l'assurance automobile du Québec (SAAQ) ( $n=1)$, lors de coupures de personnel ou de non-remplacements lors de congés $(n=5)$ ou peuvent être occasionnés par les longues listes d'attentes $(n=4)$. Par exemple, au sujet de ce dernier élément, l'ergothérapeute $n^{\circ} 5$ rapporte qu'au sein de son Centre de santé et de services sociaux (CSSS), les soins offerts aux personnes ayant un problème de santé mentale et étant âgées de moins de 65 ans sont offerts par l'équipe de santé mentale, alors qu'ils sont prodigués par l'équipe du soutien à domicile lorsque les personnes atteignent l'âge de 65 ans. Cela dit, étant donné que les listes d'attente du programme de soutien à domicile sont longues, que les cas de santé mentale ne sont pas priorisés au programme de soutien à domicile et que le nombre de professionnels desservant cette clientèle n'est pas déterminé au prorata des personnes qui nécessitent des soins, ces personnes vivent bien souvent des vides de services, c'est-à-dire qu'ils n'ont au final accès aux soins qu'après des délais d'attente très grands. Ils peuvent également vivre une cessation de services advenant le cas où ils étaient suivis par l'équipe de santé mentale jusqu'à l'âge de 65 ans. Les critères des programmes de soutien à domicile, dans ce cas, sont déterminés d'après l'impact des incapacités physiques des personnes sur leur fonctionnement à domicile, alors que les incapacités reliées à la santé mentale de ces personnes peuvent elles aussi avoir un très grand impact négatif sur leur fonctionnement. Les critères actuellement utilisés afin d'établir la priorisation ou de déterminer le niveau d'urgence ne semblent pas prendre en compte cette réalité pour l'instant, selon cette participante.

Aussi, selon les ergothérapeutes rencontrées, la complexité du problème de santé de ces personnes fait en sorte que certains membres des équipes interdisciplinaires, dont font partie les ergothérapeutes, n'ont pas la même vision de ce qui doit être fait auprès de cette clientèle, ce qui rend difficile l'harmonisation des soins pour ces individus qui présentent des besoins multiples $(n=4)$. Autrement dit, les ergothérapeutes, œuvrant au sein de différents milieux de soins et en équipe avec des membres de diverses professions, peuvent être confrontés à des paradigmes et à des valeurs 
professionnelles parfois différents des leurs et à des zones grises concernant ce qui doit être fait et par quel professionnel. L'ergothérapeute $n^{\circ} 2$ explique cette situation ainsi :

C'est sûr que ce n'est pas tout le monde qui a une vision holistique. Même si tu mets les meilleures recommandations au monde avec ta vision holistique, cela ne veut pas dire que les gens qui vont les appliquer l'ont. Cela ne veut pas dire que les gens sont aussi motivés que toi ou qu'ils ont la même compréhension que toi du cas.

De plus, la majorité des ergothérapeutes rencontrées $(n=4)$ ont exprimé que certains préjugés entourant les troubles de santé mentale demeurent dans les milieux de soins, ce qui influence négativement les trajectoires de soins de personnes présentant à la fois un problème relevant du domaine de la santé physique et un autre de la santé mentale. Lorsqu'elles discutent de cet élément, les ergothérapeutes interrogées affirment avoir ici leur part de responsabilités. En ce sens, il leur arrive de participer à certaines ruptures ou cessations précoces de soins, par leur anticipation de la complexité de certains cas ou leur méconnaissance de certains problèmes de santé mentale. Pour illustrer cette affirmation, voici ce que l'ergothérapeute $n^{\circ} 5$ exprime à ce sujet :

Quand on voit cela [le diagnostic de santé mentale au dossier], on n'est pas toujours content. On anticipe le problème et la complexité. On se dit que cela va être un dossier difficile. On ne se prépare pas toujours positivement. On a parfois un préjugé défavorable à la lecture du diagnostic, par rapport à quelqu'un qui n'aurait pas le même diagnostic et une demande similaire.

Dans le même ordre d'idées, trois ergothérapeutes ont mentionné que les personnes présentant une problématique complexe de santé sont plus à risque de voir leurs soins interrompus ou écourtés lorsqu'ils cheminent dans le RSSS, comparativement à d'autres clientèles. L'ergothérapeute $n^{\circ} 4$ avoue ceci: "Les ergothérapeutes, c'est quoi notre occupation? C'est d'être ergothérapeute. Alors, qu'est-ce qui est signifiant pour nous? Nous aussi on va faire notre travail pour rechercher le plaisir, pour rendre cela agréable. C'est sûr qu'on va éliminer les obstacles... " II arrive donc que les soins des clients considérés plus lourds soient interrompus ou écourtés, en raison de la complexité, voire de la perception défavorable de la complexité des cas, selon des participantes.

Par la suite, trois ergothérapeutes ont affirmé que les critères d'admission des différents programmes de santé sont parfois très stricts ou discriminatoires, ce qui contribue au manque d'intégration horizontale des soins ainsi qu'à la difficulté d'accès aux soins pour plusieurs personnes. Par exemple, dans certains milieux, une personne qui présente un trouble anxieux et qui n'est pas en mesure de réaliser seule ses soins d'hygiène (peur de tomber, anxiété de ne pas être capable de se relever advenant une chute, désorganisation trop grande due à l'anxiété) est susceptible de ne pas pouvoir recevoir d'aide à l'hygiène de la part du Centre local de services communautaires (CLSC) de sa région, étant donné qu'elle a les capacités physiques nécessaires pour réaliser cette occupation et que les critères déterminants la possibilité de recevoir de l'aide à l'hygiène sont centrés uniquement sur ces capacités physiques (amplitudes articulaires, force musculaire, niveau d'énergie, équilibre, etc.). Cette réalité est exprimée clairement par l'ergothérapeute $n^{\circ} 5$ : "Tout le monde resserre les critères. On resserre l'accès aux services. On établit des cadres. C'est difficile d'avoir accès. C'est difficile pour la clientèle. Les demandes se promènent de bureau en bureau. Ce n'est pas tellement dans les mains des intervenants que cela se passe. "Bref, ce n'est pas seulement le manque de continuité dans les trajectoires de soins qui est ici pointé du doigt, mais également l'accessibilité aux soins.

Enfin, trois ergothérapeutes $(n=3)$ discutent des pressions de rendement et de performance qu'ils ressentent dans leur travail au quotidien, qui expliquerait d'après elles, en partie, les discontinuités dans les trajectoires de soins que vivent ces personnes. L'ergothérapeute $n^{\circ} 5$ explique que ces pressions relatives à l'optimisation des soins nuisent en quelque sorte à sa pratique idéale de 
l'ergothérapie: " Je n'ai pas beaucoup de temps et de liberté de mon employeur d'aborder toutes les sphères [de la personne]. On n'aime pas non plus se faire juger sur son rendement. Tu es toujours pris entre les demandes des clients et l'utopie de la profession ». Les ergothérapeutes rencontrées ont la perception que le contexte organisationnel actuel privilégie presque uniquement le rendement et la performance. Bien qu'il soit sous-entendu qu'il importe également d'offrir des soins de qualité, elles ont le sentiment que les diktats relatifs au rendement et à la performance occultent les nombreux autres aspects de la pratique qui peuvent expliquer que les soins offerts aux clients soient de facto des soins de qualité. Cela se manifeste plus particulièrement, selon elles, par le fait que les critères pour évaluer les soins sont majoritairement de nature quantitative (aspects financiers des soins, nombre de personnes desservies, temps des interventions directes et indirectes, etc.) et trop peu de nature qualitative (satisfaction de la clientèle, perception de la qualité des soins reçus, perception d'avoir reçu des soins spécifiques à sa condition, etc.). L’ergothérapeute $n^{\circ} 5$ explique cette réalité ainsi:

Actuellement, on a des consignes claires d'accélérer les processus d'intervention : de voir plus de clients par jour, de voir plus de clients par semaine. On ne peut pas accorder le temps qu'on souhaiterait à chaque type de demande. On a des comptes à rendre sur le nombre de visites qu'on a faites. On est évalué sur le rendement, sur la vitesse, sur la quantité. Pour toutes les sphères occupationnelles, on pourrait tellement faire plus, si c'était reconnu et si on avait la liberté de le faire.

Selon cette participante, le manque de temps a définitivement un impact négatif sur la clientèle et les soins qu'elle reçoit. L'ergothérapeute $n^{\circ} 5$ ajoute : "Cela prend du temps; cela prend l'énergie. II faut vouloir accueillir la personne avec ses difficultés, mais dans le contexte actuel on n'a pas beaucoup le temps, cela nous surcharge tous d'absorber cela. On dirait qu'on cherche à atteindre nos objectifs et à fermer le dossier ». Bref, les processus d'optimisation des soins qui ont cours dans le RSSS, créant une pression de rendement et de performance ressentie par les intervenants, contribuent à expliquer les discontinuités dans les trajectoires de soins vécues par les individus ayant une problématique complexe de santé, selon plusieurs des ergothérapeutes interrogées.

\section{Conséquences des discontinuités dans les trajectoires de soins}

Comme l'indique le Tableau 3, en plus des raisons qui contribuent à expliquer les discontinuités dans les trajectoires de soins, les participantes ont discuté des conséquences sur les clients et les ergothérapeutes qu'occasionnent ces ruptures lorsqu'elles ont été questionnées à ce propos.

\section{Tableau 3 : Principales conséquences des discontinuités dans les trajectoires des soins}

\begin{tabular}{|l|c|}
\hline Conséquences des discontinuités & $\begin{array}{c}\text { Nombre } \\
\text { d'ergothérapeutes }\end{array}$ \\
\hline $\begin{array}{l}\text { Nuit à la réponse adéquate à la multiplicité des besoins des } \\
\text { clients }\end{array}$ & 5 \\
\hline Suscite des enjeux éthiques importants chez les ergothérapeutes & 5 \\
\hline
\end{tabular}

D'abord, toutes les participantes ont exprimé que les discontinuités dans les trajectoires de soins nuisent à la réponse adéquate à la multiplicité des besoins de ces personnes. C'est comme si tout était à recommencer chaque fois qu'une discontinuité dans la prestation des soins se présentait. Par exemple, celles-ci font en sorte que les évaluations se répètent au détriment des traitements (ergothérapeute $n^{\circ} 5$ ). Étant donné que les temps d'intervention sont parfois écourtés dans le contexte actuel et que les interventions débutent par une évaluation, le client se trouve parfois à répéter son histoire personnelle et à subir de nouveau les mêmes évaluations, au détriment d'interventions qui lui seraient utiles pour contrer les conséquences négatives de ses problèmes de santé. Somme toute, selon les participantes, les ruptures ont un impact négatif sur la qualité des interventions offertes à ces personnes et d'autant plus dans le contexte actuel où les pressions relatives à l'optimisation ne 
cessent de croître. II faut aller vite, produire plus avec moins : la conséquence en est que la qualité des soins en écope, estiment-elles. Plus encore, les participantes ont la perception que les interventions sont moins efficaces, en ceci que les problèmes de santé de ces personnes persistent, comparativement aux difficultés des individus qui vivent un problème de santé moins complexe. Par exemple, l'ergothérapeute $n^{\circ} 4$ dit ceci : "Cela a nui à son rendement occupationnel [au rendement du client] parce qu'on ne s'est pas occupé de la partie physique découlant de la problématique de santé mentale ». Puis, l'ergothérapeute $\mathrm{n}^{\circ} 1$ ajoute cet élément : "Elle [la cliente] est sûrement restée avec des limitations. Les recommandations ne sont pas généralisées à la maison. Cela a un impact partout dans les habitudes de vie ". En somme, les participantes considèrent que les interventions offertes ont peu d'impact sur le fonctionnement des personnes recevant des soins $(n=4)$ et ne sont pas adaptées à la situation unique de chacun.

En plus de cela, toutes les participantes ont identifié que les situations de discontinuités dans les trajectoires de soins de personnes présentant une problématique complexe de santé les confrontent davantage que d'autres situations et soulèvent des enjeux éthiques au quotidien, notamment des enjeux relatifs à la micro-allocation des soins. L'enjeu éthique qui a été rapporté par la majorité des participantes est que ce type de clientèle demande un investissement important, notamment en termes de temps d'intervention. Or, lorsqu'elles constatent le temps investi et le peu de résultats cliniques tangibles, elles se questionnent quant à savoir si d'autres clients n'auraient pas pu bénéficier davantage de ce temps investi et s'il était approprié d'en faire autant étant donné la faible efficacité des interventions. Les propos de l'ergothérapeute $\mathrm{n}^{\circ} 3$ illustrent ces questionnements relatifs à la justice distributive dans sa pratique : "C'est comme s'ils arrivent [les clients présentant une problématique complexe de santé] et que leurs besoins sont dans toutes les sphères. Mais on a d'autres clients en même temps, on ne peut pas... J'aurais pu passer la semaine pour ce client-là ». L'ergothérapeute $\mathrm{n}^{\circ} 1$ renchérit en disant : "Ces dossiers-là sont rarement des réussites, il n'y a pas d'aboutissements. " Comment, dans ce contexte, distribuer les soins de manière équitable tout en répondant aux besoins des personnes, se demandent les participantes?

Aussi, les ergothérapeutes constatent que cette clientèle est plus à risque d'avoir de la difficulté à défendre ses droits que d'autres clientèles. Devant la complexité du système de santé et leur vulnérabilité relative, les ergothérapeutes estiment qu'elles ont un rôle à jouer auprès de cette clientèle. Or, ce rôle demande du temps. L'ergothérapeute $n^{\circ} 4$ explique le problème sous-jacent à la vulnérabilité que présentent certains clients : "Une personne qui n'a plus l'aptitude de défendre ses droits, dans un endroit où on coupe, on va défendre la personne qui parle et se défend. Celle qui ne se défend pas, on va penser qu'on l'a desservie... " Comment défendre de manière équitable les droits des clients? Comment le faire dans le contexte actuel où tout va vite et où le temps manque? Les participantes considèrent qu'elles ont une responsabilité professionnelle relative à l'advocacy ${ }^{11}$ eu égard à cette clientèle. Cette responsabilité s'appuie sur une certaine vision qu'elles ont de leur profession, sur une certaine éthique professionnelle. En bref, l'intervention auprès de cette clientèle confronte les participantes à des enjeux éthiques qu'elles estiment importants.

En résumé, les participantes sont partagées : elles constatent, d'une part, les nombreux besoins de ces personnes et leurs difficultés à défendre par elles-mêmes leurs droits et se sentent conséquemment interpelées pour les aider, mais réalisent, d'autre part, que les succès cliniques ne sont pas fréquents avec elles et que le temps investi est très élevé comparé à d'autres clientèles, ce qui peut contribuer à les démotiver dans leur désir de s'investir auprès de ces personnes.

\section{Pistes de solution proposées par les participantes}

Les questions posées aux participantes leur ont aussi permis de discuter de pistes de solution qui pourraient être appliquées par différents acteurs ou partenaires du RSSS, dans le but de réduire les

\footnotetext{
${ }^{11}$ Il est difficile de traduire le mot advocacy, car ce terme réfère à plusieurs réalités comme la défense des droits des clients, la revendication au nom de leurs besoins et intérêts ainsi que la promotion de la profession. Étant donné qu'il couvre diverses réalités et qu'il est abondamment utilisé en français sans qu'il soit traduit, nous avons décidé de ne pas le traduire.
} 
iniquités relatives à la continuité et à l'accessibilité des soins offerts aux personnes présentant une problématique complexe de santé. Comme l'indique le Tableau 4, les recommandations formulées par les participantes concernent les décideurs, les ergothérapeutes (ou, de façon plus globale, les intervenants du réseau de la santé) ou les clients.

Tableau 4 : Pistes de solution proposées par les participantes

\begin{tabular}{|l|l|c|}
\hline Pistes de solution & \multicolumn{1}{|c|}{ Acteurs ciblés } & $\begin{array}{c}\text { Nombre } \\
\text { d'ergothérapeutes }\end{array}$ \\
\hline $\begin{array}{l}\text { Mise en place de plateformes collaboratives entre les } \\
\text { établissements }\end{array}$ & Décideurs du RSSS & 5 \\
\hline $\begin{array}{l}\text { Développement des qualités professionnelles et } \\
\text { personnelles de l'ergothérapeute }\end{array}$ & Ergothérapeutes & 5 \\
\hline Véritable travail collaboratif et interdisciplinaire & $\begin{array}{l}\text { Ergothérapeutes ou autres } \\
\text { intervenants }\end{array}$ & 4 \\
\hline $\begin{array}{l}\text { Accès à plus de formations pour gérer les problèmes de } \\
\text { santé mentale }\end{array}$ & Décideurs du RSSS & 3 \\
\hline $\begin{array}{l}\text { Valorisation de l'holisme dans les interventions } \\
\text { Advocacy au nom et en collaboration avec le client pour la } \\
\text { défense de ses droits et promotion de la profession } \\
\text { d'ergothérapeute }\end{array}$ & $\begin{array}{l}\text { Ergothérapeutes ou autres } \\
\text { intervenants }\end{array}$ & Ergothérapeutes et clients \\
\hline
\end{tabular}

D'abord, certaines pistes de solution proposées par les participantes s'adressent aux principaux décideurs du RSSS. Par exemple, toutes les ergothérapeutes rencontrées ont revendiqué, d'une part, la mise en place de plateformes de soins véritablement collaboratives entre les différents milieux de soins pour améliorer les communications entre les intervenants des établissements de santé physique et ceux de santé mentale, ce qui inclut le milieu communautaire et, d'autre part, l'accès à plus de formations portant sur les meilleures façons de gérer les problèmes de santé mentale $(n=3)$. Ensuite, certaines pistes de solution proposées par les participantes sont directement liées à la pratique des intervenants. Toutes les participantes ont exprimé qu'il est nécessaire que les ergothérapeutes développent certaines qualités professionnelles et personnelles pour faciliter les trajectoires de soins des personnes présentant une problématique complexe de santé, par exemple être de nature proactive, avoir une ouverture et une sensibilité à l'autre et avoir le souci d'offrir des soins de qualité.

En plus de ces éléments, la majorité des participantes rencontrées $(n=4)$ identifient que le travail en équipe interdisciplinaire et le réseautage intra et interprofessionnel sont de grands facilitateurs pour assurer la continuité des soins et la réponse adéquate aux besoins des personnes présentant une problématique complexe de santé. Pour illustrer cela, l'ergothérapeute $n^{\circ} 4$ explique ceci : « Je pense que le réseautage est essentiel. Le réseautage, ce n'est pas nécessairement entre ergothérapeutes. Dans une équipe, aussi. Mais c'est bien de discuter entre ergothérapeutes ». Également, une autre solution proposée par les ergothérapeutes rencontrées $(n=3)$ est que l'ergothérapeute est un professionnel qui peut agir à titre d'agent intégrateur des soins et services, de par son approche globale et sa vision holistique de la personne. La majorité des participantes à l'étude mentionnent que l'application de l'approche holistique de la personne, plus particulièrement dans l'évaluation en ergothérapie, est une piste de solution riche étant donné qu'elle permet de dépister les difficultés que vivent les personnes dans les différentes sphères qui les composent (santé physique, santé mentale, santé sociale, etc.). Pour l'ergothérapeute $\mathrm{n}^{\circ} 2$, l'approche holistique est un outil qui permet aux ergothérapeutes d'agir à titre d'intervenant pivot auprès de ce type de clientèle : "Je suis vraiment l'intervenante pivot dans des dossiers comme cela, donc le rôle de l'ergo est assez important auprès des personnes qui ont des doubles ou des triples problématiques ". II appert toutefois que le rôle d'intervenant pivot est parfois retiré aux ergothérapeutes dans certains milieux cliniques, malgré qu'il puisse s'avérer pertinent et nécessaire que ce soit ce professionnel qui occupe ce rôle. Une certaine 
flexibilité ou révision des façons actuelles de faire, passant par la sensibilisation des autres membres de l'équipe à la vision holistique de la personne ou par une manifestation de l'intérêt de l'ergothérapeute à agir à titre d'intervenant pivot dans certains cas, pourrait aider à harmoniser les soins pour certaines personnes, estiment les participantes. L'ergothérapeute $n^{\circ} 5$ soutient cette perception de la sorte : " Je pense qu'on serait les mieux placées. On développe beaucoup dans les soins et services, on devrait peut-être regarder l'approche en ergothérapie où on évalue l'ensemble des dimensions et où on cerne ce qui fait qu'il y a un impact sur l'autonomie. [...] Je pense qu'on aurait intérêt à être beaucoup plus présentes. " En somme, pour plusieurs participantes, la valeur qu'est l'holisme est un atout pour intervenir auprès des personnes ayant une problématique complexe de santé, ce qui explique, selon elles, qu'elles sont habilitées à être l'intervenante pivot au sein de l'équipe.

Enfin, deux participantes à l'étude ont exprimé qu'il est important et même nécessaire que l'ergothérapeute ait les habiletés pour plaidoyer au nom du client et pour l'outiller à défendre par luimême ses droits. L'ergothérapeute $n^{\circ} 4$ explique que d'établir un partenariat ergothérapeute-client est nécessaire pour aider ceux-ci à être correctement représentés :

Je pense que c'est important de créer des alliances, de favoriser le partenariat, de plaidoyer pour son client et de s'organiser pour que lui aussi il puisse plaidoyer pour sa cause. Si on sent qu'on pourrait répondre ensemble à son besoin et que moi j'ai des limites dans ma façon d'argumenter, c'est au client à faire valoir ses droits. [...] II faut accompagner la personne à faire valoir ses droits. Une façon de plaidoyer pour le client, c'est aussi de l'aider à plaidoyer pour lui-même.

Cette valorisation de l'advocacy se manifeste également sous d'autres formes, soit dans l'importance qu'accordent les ergothérapeutes au fait de mettre à profit leur rôle de communicateur et d'agent de changement, en vue de plaidoyer ou de revendiquer pour les clients, mais aussi de promouvoir la profession au sein de comités ou d'instances administratives. Au sujet de ce dernier élément, les participantes considèrent qu'elles doivent faire valoir qu'elles sont des professionnelles de la santé ayant des habiletés pouvant faciliter les trajectoires de soins des personnes présentant une problématique complexe de santé, d'où la pertinence de plaidoyer pour la profession dans ces cas.

\section{Discussion}

\section{Questions et objectifs de la recherche}

La question à l'origine de cette étude exploratoire était la suivante : Quelles sont les perceptions d'ergothérapeutes du continuum de soins et, s'il y en a, des discontinuités dans les trajectoires de soins que vivent des personnes ayant une problématique complexe de santé? II s'ensuit que l'objectif de la recherche était d'explorer les perceptions d'ergothérapeutes de la continuité, de la discontinuité et des vides de services que rencontrent des clients ayant une concomitance entre au moins un problème de santé physique et au moins un problème de santé mentale. Comme spécifié antérieurement, des entretiens individuels ont été réalisés avec cinq ergothérapeutes afin d'apporter des pistes de réponses à cette question et d'atteindre cet objectif. La section précédente présente un résumé des perceptions des participantes à l'étude des discontinuités dans les trajectoires de soins de personnes ayant une problématique de santé complexe selon trois thèmes : les raisons de ces ruptures, leurs conséquences ainsi que les pistes de solutions proposées par les participantes. De fait, les perceptions d'ergothérapeutes desservant des clients ayant une problématique complexe de santé ont été explorées.

\section{Interprétations des résultats}

Quelles conclusions peut-on tirer des résultats? Quelles interprétations peut-on en faire? D'abord, il est intéressant de constater que n'eut égard au fait que les cinq participantes à l'étude œuvraient au 
sein de milieux de pratique différents, étaient d'âges variés et avaient un nombre d'années d'expérience différent, toutes ont identifié des situations où des personnes présentant une problématique complexe de santé avaient vécu des discontinuités dans leurs trajectoires de soins. Selon elles, ces individus étaient plus susceptibles que d'autres clients de vivre de tels épisodes, lesquels ne correspondent pas à un idéal de pratique, notamment parce que ces ruptures ont des conséquences négatives à la fois pour les clients (affectent négativement la qualité des soins) et pour elles-mêmes (engendrent des enjeux éthiques importants).

Ensuite, un examen des raisons qui, d'après les ergothérapeutes rencontrées, expliquent ces discontinuités dans les trajectoires de soins de ces clients montre que leurs causes sont multiples et de nature variée. Selon elles, une seule cause est relative à la clientèle elle-même (la complexité de leur problème de santé), trois ont trait aux intervenants (les visions distinctes de l'être humain dans l'équipe, les préjugés des intervenants relatifs à la santé mentale ainsi que la pression de rendement et d'efficacité ressentie par les intervenants) et cinq concernent l'organisation des soins (cloisonnement des soins, suppressions de postes et non-remplacements des congés, processus d'optimisation, vides de services et critères d'admission stricts des établissements). Ainsi, les ergothérapeutes considèrent que les causes de ces ruptures seraient pour la plupart de nature organisationnelle et dans une moindre mesure, reliées aux personnes et à leurs interactions. II en est de même lorsqu'elles proposent des solutions afin de corriger ces situations. Les solutions envisagées concernent pour la plupart l'organisation des soins, voire l'environnement au sens large (mise en place de plateformes collaboratives entre les établissements, véritable travail collaboratif et en interdisciplinarité, accès à plus de formations pour gérer les problèmes de santé mentale et valorisation de l'holisme dans les interventions), sans négliger toutefois leurs responsabilités éthiques individuelles (développement des qualités professionnelles et personnelles de l'ergothérapeute ainsi que du devoir d'advocacy au nom et en collaboration avec le client pour la défense de ses droits ou pour promouvoir la profession d'ergothérapeute et sa vision holistique). Cette considération des dimensions à la fois environnementales et personnelles par les ergothérapeutes rencontrées n'est pas étonnante. II faut dire que les modèles conceptuels utilisés par les ergothérapeutes comme le Modèle Personne-Environnement-Occupation [37] ou le Modèle canadien du rendement et de l'engagement occupationnel [22] comprennent les dimensions environnementales et personnelles pour analyser l'autonomie des personnes et, par extension, leurs occupations.

De plus, les discours des ergothérapeutes rejoignent des préoccupations éthiques de certains philosophes contemporains. À titre d'exemple, à la manière de Martha Nussbaum qui, dans son éthique des capabilités, prend en compte les dimensions sociétales pour appréhender le respect des droits des personnes, les ergothérapeutes rencontrées ne négligent pas ces considérations systémiques qui de fait affectent les vies concrètes des personnes, notamment celles des personnes vulnérables [38]. Aussi, à la manière de maints utilitaristes contemporains comme Peter Singer, les participantes-ergothérapeutes considèrent les conséquences que l'organisation actuelle des soins a sur les principaux acteurs de la situation, en l'occurrence les clients et les intervenants [39]. De plus, suivant les déontologues comme John Rawls, elles constatent les iniquités de droits qui existent entre les clients et s'en désolent [40]. Ce faisant, elles envisagent des solutions afin de corriger ces situations qui, espèrent-elles, pourront favoriser le respect des droits de chacun, en particulier des clientèles vulnérables qui présentent une problématique complexe de santé. Par la suite, à la manière de certains éthiciens de la vertu qui s'inspirent du philosophe Aristote, les ergothérapeutes ne négligent pas non plus l'importance du développement de leurs qualités à la fois professionnelles et personnelles, recherchant de la sorte une certaine excellence dans leur pratique [41]. Aussi, la vertu qu'est la sollicitude pour les personnes ayant une problématique complexe de santé ressort clairement de leurs propos. Enfin, elles discutent d'une valeur qui leur est chère, soit l'holisme, puisant ainsi aux sources axiologiques de leur profession. De fait, la vision holiste de l'ergothérapeute est considérée par les participantes comme un atout qui pourrait permettre de penser autrement les soins et de mieux répondre aux besoins des clients, notamment ceux ayant une problématique complexe de santé. En bref, lorsque l'on examine les résultats par l'entremise du Cadre éthique 
quadripartite [42,43], qui consiste en un cadre d'analyse éthique comprenant quatre fenêtres pour conduire des analyses éthiques de situations problématiques sur le plan de l'éthique, il en résulte que les participantes ont des préoccupations éthiques de nature utilitariste et déontologique, de même que des préoccupations apparentées à l'éthique des vertus et d'autres, liées aux valeurs aux assises de leur profession.

\section{Comparaisons entre les résultats et certains écrits}

Comme documenté dans les écrits [5,8,10-12], les personnes présentant une problématique de santé complexe vivent des discontinuités dans leurs trajectoires de soins, comme l'ont également noté les participantes à l'étude. Plus encore, certaines de ces personnes sont confrontées à des difficultés d'accès à des soins et même à des vides de services [9], comme l'ont aussi noté les participantes. Par exemple, un client ayant une déficience physique (comme une hémiplégie, une lombalgie, une blessure médullaire ou une amputation) associée à un trouble de santé mentale (comme une dépression, une schizophrénie, une bipolarité ou un autre trouble de la personnalité) désirant retourner au travail pourrait avoir de la difficulté à recevoir des soins de réadaptation au travail qui répondent à l'ensemble de ses besoins comme rapporté au début de cet article.

Un autre élément qui est ressorti de cette étude et qui s'arrime avec les écrits est le fait que la notion de comorbidité était équivoque pour certaines participantes [3]. De fait, des participantes avaient des définitions variables de ce concept. À ce sujet, les ergothérapeutes rencontrées qui travaillaient plus spécifiquement en santé mentale usaient de ce terme en référant parfois à des personnes ayant un problème de santé mentale associé à une démence, une déficience intellectuelle ou un problème de toxicomanie par exemple. Précisons que le terme " comorbidité » est aussi utilisé dans les écrits pour référer à ces personnes [14].

De plus, comme suggéré par Williams et ses collaborateurs [11,12], la valeur qu'est l'holisme est considérée par maints ergothérapeutes comme un atout important pour la réponse adéquate et continue aux besoins multiples des clients ayant une problématique complexe de santé. Le paradigme biomédical ne permettrait pas de rendre compte de l'ensemble des besoins de ces personnes, alors qu'une approche holiste serait davantage en mesure d'y parvenir, comme le suggèrent aussi les participantes. Le fait de concevoir l'être humain comme un tout indissociable et d'avoir ainsi une vision holiste de la personne facilite la prise en compte des besoins à la fois physiques et psychologiques des personnes. Comme l'estiment Bjorklund, Svensson et Read [16], l'holisme se présente comme une solution pour concevoir une organisation et une prestation différente de soins de santé, lesquelles seraient plus aptes à répondre aux besoins des personnes. Cela dit, encore faut-il que cette valeur soit actualisée, pas seulement idéalisée, et que chacun en ait une même représentation [20].

Un autre élément discuté par plusieurs participantes est également présent dans les écrits, soit l'importance de soutenir ces clientèles, par ailleurs vulnérables, dans la revendication et la défense de leurs droits [43-55]. Pour maintes ergothérapeutes rencontrées, cette idée va de soi : elles ont le devoir de soutenir ces clients dans la promotion et la défense de leurs droits, intérêts et besoins. En tant qu'agentes de changement, l'une des sept compétences de l'ergothérapeute selon le Profil de la pratique des ergothérapeutes au Canada [44], elles affirment faire des actions en ce sens, en collaboration avec les clients concernés et auprès de divers tiers ou instances. Cela dit, la pratique de l'advocacy effectuée par les ergothérapeutes commence à peine à être documentée empiriquement [56].

Certaines participantes ont également affirmé que certains critères d'inclusion ou d'exclusion à des programmes ou à des services étaient basés sur des critères quantitatifs ou étaient trop strictes et rigides, pouvant même aller jusqu'à exclure certaines personnes vivant des problématiques complexes de santé. Elles estiment même que certains de ces critères sont discriminatoires. Par exemple, dans certains CSSS, une personne en perte d'autonomie nécessitant une adaptation de son 
domicile due à son vieillissement et souffrant d'un trouble psychotique nécessitant un suivi par une équipe en santé mentale pourrait, dans certains cas, ne recevoir qu'un seul des deux services (service concernant sa condition physique ou service concernant sa condition psychiatrique). En effet, dans ce cas-ci, cette personne serait desservie par l'équipe en santé mentale avant qu'elle n'atteigne l'âge de 65 ans, pour qui la majeure préoccupation concerne la condition psychiatrique de la personne. Cette même personne serait desservie par l'équipe de soutien à domicile lorsqu'elle atteindrait l'âge de 65 ans, la condition psychiatrique étant davantage laissée de côté à ce moment et la majeure préoccupation concernant dorénavant la condition physique de la personne. Cela occasionne généralement des discontinuités et parfois des vides de services lors de l'atteinte de l'âge de 65 ans, puisque le suivi en santé mentale est cessé et que la personne est habituellement inscrite sur une liste d'attente au programme de soutien à domicile. Dans la même veine, Schwartz estime que le fait de fonctionner en silo ou de diviser les personnes en catégories ne permet pas d'accueillir les histoires singulières ou les problématiques complexes des individus et, par le fait même, de répondre avec diligence à l'entièreté de leurs besoins [57].

Enfin, les propos des ergothérapeutes rejoignent les préoccupations ministérielles relatives à une meilleure intégration verticale et horizontale des soins afin d'offrir une réponse optimale à la multiplicité des besoins de santé des personnes [5]. Elles estiment aussi qu'une offre de services complémentaires, des partenariats et des collaborations efficaces sont nécessaires afin d'améliorer l'accès, la qualité et la globalité des soins. Cela dit, leur compréhension de ce problème sociétale va plus loin. Elle prend aussi en compte des éléments plus fondamentaux. Les ergothérapeutes considèrent que la séparation entre, d'une part, les établissements répondant aux problèmes de santé physique des personnes et, d'autre part, d'autres établissements procurant des soins de santé mentale participe en quelque sorte à une vision morcelée des individus et concourt en ce sens à une réponse plus ou moins adéquate à leurs besoins. Il en découle que les changements à l'organisation des soins qui ne considèrent pas cet élément sont en quelque sorte incomplets.

\section{Forces et limites de la recherche}

La force principale de cette étude a trait à sa pertinence. Bien qu'exploratoire, cette étude est pertinente à la fois d'un point de vue scientifique, professionnel et social. Scientifiquement, les perceptions d'ergothérapeutes quant à ce problème sociétal n'ont pas, à ce jour, été documentées. Professionnellement, elle peut permettre à des ergothérapeutes de se reconnaître dans les propos des ergothérapeutes rencontrées et entreprendre des démarches pour mettre de l'avant certaines des solutions envisagées et promouvoir le changement vers l'application de celles-ci. Socialement, elle vise à mieux desservir les personnes ayant une problématique complexe de santé et, pour y parvenir, il importe de documenter leurs situations, ce que fait indirectement cette recherche, bien que modestement. Ce faisant, cette recherche exploratoire soulève des situations à corriger à l'avenir et cible différentes avenues afin de corriger ces lacunes qui ont des conséquences négatives sur les personnes ayant une problématique complexe de santé et sur les intervenants, notamment les ergothérapeutes. Ainsi, en plus de pointer des aspects problématiques de la situation actuelle qui doivent être repérés et clairement ciblés, cette recherche propose des pistes de solutions visant à corriger, en partie du moins, la situation en vue d'une prestation plus souhaitable des soins et davantage en conformité avec les actuelles politiques ministérielles.

Par contre, cette recherche présente certaines limites. D'abord, sur le plan de l'échantillonnage, le nombre peu élevé de participants limite la transférabilité des résultats; ceux-ci doivent donc être utilisés avec prudence. La saturation des données n'ayant pas été atteinte, il est possible que le phénomène n'ait été cerné que partiellement. Toutefois, même si cette recherche était exploratoire, elle montre que le phénomène méritait d'être étudié et, en ce sens, d'autres recherches pourraient poursuivre ce premier pas certes modeste, mais pointant un problème sociétal préoccupant d'un point de vue éthique. II est en effet préoccupant que des personnes vulnérables n'aient pas accès à des soins qui répondent à l'ensemble de leurs besoins, et ce, sans discontinuités dans leurs trajectoires de soins ni vides de services. 


\section{Conclusion}

Cette étude a exploré les perceptions d'ergothérapeutes au sujet de la continuité et de la discontinuité dans les trajectoires de soins des personnes présentant une problématique complexe de santé. Les participantes-ergothérapeutes ont nommé des raisons qui à leur avis expliquent les discontinuités dans les trajectoires de soins de ces personnes. Elles ont discuté des conséquences négatives que celles-ci occasionnent sur les clients et sur les intervenants, dont elles-mêmes. Elles ont proposé des solutions qui, d'après elles, pourraient minimiser, dans une certaine mesure, ces ruptures de soins. Les résultats de cette étude rejoignent en général ceux des écrits sur le sujet, de même que les préoccupations éthiques de certains philosophes contemporains. Ils s'accordent aussi avec les souhaits du Ministère de la Santé et des services sociaux du Québec quant à l'amélioration de l'intégration verticale et horizontale des soins. Cela dit, d'autres recherches documentant la situation des personnes présentant une problématique complexe de santé mériteraient d'être réalisées auprès d'un plus grand nombre de participants et auprès de participants variés (intervenants, décideurs, clients) compte tenu de la vulnérabilité de ces personnes et de leurs difficultés à avoir actuellement accès à des soins optimaux et en continu qui répondent à l'entièreté de leurs besoins.

\section{Références}

1. Desrosiers G. The Quebec health care system. Journal of Public Health Policy. 1986; 7(2): 211-217.

2. Pigeon M. La santé au Québec dans la seconde moitié du 20 e siècle. Musée McCord; 2011.

3. Buck HG, Meghani S, Bettger JP, Byun E, Fachko MJ, O'Connor M, Tocchi C, Naylor M. The use of comorbidities among adults experiencing care transitions: a systematic review and evolutionary analysis of empirical literature. Chronic Illness. 2012; 8(4): 278-295.

4. St-Pierre MA, Gouvernement du Québec. Regards sur le système de santé et de services sociaux du Québec. Québec, Santé et services sociaux Québec; 2009.

5. Gouvernement du Québec. L'intégration des services de santé et des services sociaux: Le projet organisationnel et clinique et les balises associées à la mise en œuvre des réseaux locaux de services de santé et de services sociaux. Québec, Le Ministère, Direction des communications; 2004.

6. Contandriopoulos AP, Denis JL, Touati N. Intégration des soins : dimensions et mise en œuvre. Ruptures, revue transdisciplinaire en santé. 2001; 8(2): 38-52.

7. Rondeau-Boulanger É. Perceptions d'ergothérapeutes cliniciens et de personnes aux prises avec une problématique complexe de santé des situations de ruptures dans la trajectoire de soins et de services : réflexion critique et analyse des enjeux éthiques. Essai critique dans le cadre d'une maîtrise professionnelle. Trois-Rivières: Université du Québec à Trois-Rivières; 2015.

8. Gulliford M, Cowie L, Morgan M. Relational and management continuity survey in patients with multiple long-term conditions. Journal of Health Services Research \& Policy. 2011; 16(2): 6774.

9. Tarrant C, Windridge K, Baker R, Freeman G, Boulton M. 'Falling through gaps': primary care patients' accounts of breakdowns in experienced continuity of care. Family Practice. 2015; 32(1) : 82-87.

10. Williams A, Botti M. Issues concerning the on-going care of patients with comorbidities in acute care and post-discharge in Australia: a literature review. Journal of Advanced Nursing. 2002; 40(2): 131-140.

11. Williams A. Patients with comorbidities: perceptions of acute care services. Journal of Advanced Nursing. 2004; 46(1): 13-22.

12. Williams A, Dunning T, Manias E. Continuity of care and general wellbeing of patients with comorbidities requiring joint replacement. Journal of Advanced Nursing. 2007; 57(3): 244-256.

13. Organisation Mondiale de la Santé (OMS). The World Health Report 2001 - Mental Health: New Understanding. New Hope. Geneva, World Health Organization; 2001. 
14. Organisation Mondiale de la Santé (OMS). Charge mondiale des troubles mentaux et nécessité d'une réponse globale coordonnée du secteur de la santé et des secteurs sociaux au niveau des pays. Rapport du secrétariat; 2011.

15. Clément M, Aubé D. La continuité des soins : une solution? Perspective des personnes avec comorbidité. Santé mentale au Québec. 2002; 27(2): 180-197.

16. Bjorklund A, Svensson T, Read S. Holistic and biomedical concepts of health: A study of health notions among Swedish occupational therapists and a suggestion for developing an instrument for comparative studies. Scandinavian Journal of Occupational Therapy. 2006; 13(3): 141-150.

17. Drolet MJ. The axiological ontology of occupational therapy: A philosophical analysis. Scandinavian Journal of Occupational Therapy. 2014; 21(1): 2-10.

18. Drolet MJ, Désormaux-Moreau M. Les valeurs des ergothérapeutes : Résultats quantitatifs d'une étude exploratoire. BioéthiqueOnline. 2014; 3/21.

19. Drolet MJ, Désormeaux-Moreau, M. The values of occupational therapy: perceptions of occupational therapists in Quebec. Scandinavian Journal of Occupational Therapy, Early Online, 2015:1-14.

20. Finlay L. Holism in occupational therapy: elusive fiction and ambivalent struggle. American Journal of Occupational Therapy. 2001; 55(3): 268-276.

21. Lloyd C, King R. Organisational change and occupational therapy. British Journal of Occupation Therapy. 2002; 65(12): 536-42.

22. Townsend EA, Polatajko HJ. Habiliter à l'occupation. Faire avancer la perspective ergothérapique de la santé, du bien-être et de la justice par l'occupation. Ottawa : CAOT Publications ACE; 2013.

23. West $W$. A reaffirmed philosophy and practice of occupational therapy for the 1980 s. American Journal of Occupational Therapy. 1984; 38(1): 15-23.

24. Yerxa EJ. Some implications of occupational therapy's history for its epistemology, values, and relation to medicine. American Journal of Occupational Therapy. 1992; 46(1): 79-83.

25. Descartes R. Méditations métaphysiques. Paris: GF-Flammarion;1979.

26. Swisher LL, Arsalanian LE, Davis CM. The real individual process situation (RIPS) model of ethical decision making. HPA Ressource. 2005; 5(3): 1-8.

27. Penny NH, Ewing TL, Hamid RC, Shutt KA, Walter AS. An investigation of moral distress experienced by occupational therapists. Occupational Therapy In Health Care. 2014; 28(4): 382-393.

28. Beagan B, Ells C. Values that matter, barriers that interfere: The struggle of Canadian nurses to enact their values. Canadian Journal of Nursing Research. 2009; 41(1): 86-107.

29. Kälvemark S, Höglunda AT, Hansson MG, Westerholm P, Arnetz B. Living with conflictsethical dilemmas and moral distress in the health care system. Social Science \& Medicine. 2004; 58(6): 1075-1084.

30. Edwards H, Dirette D. The relationship between professional identity and burnout among occupational therapists. Occupational Therapy in Health Care. 2010; 24(2): 119-129.

31. Gadamer HG. La philosophie herméneutique. Paris: Presses Universitaires de France; 2001.

32. Hunt MR, Carnevale FA. Moral experience: A framework for bioethics research. Journal of Medical Ethics. 2011; 37(11): 658-662.

33. Fortin F, Gagnon J. Fondements et étapes du processus de recherche: Méthodes quantitatives et qualitatives. Montréal : Chenelière éducation; 2010.

34. Fontana A, Frey J. The Art of Science. The Handbook of Qualitative Research. 1994.

35. Giorgi $A$. De la méthode phénoménologique utilisée comme mode de recherche qualitative en sciences humaines : théories, pratique et évaluation. In : J Poupart, LH Groulx, JP Deslauriers, A Lapierre, R Mayer, AP Pires (eds.). La recherche qualitative: enjeux épistémologiques et méthodologiques. Boucherville, Gaëtan Morin; 1997: 341-364.

36. Corbière $\mathrm{M}$, Larivière $\mathrm{N}$. Méthodes qualitatives, quantitatives et mixtes dans la recherche en sciences humaines, sociales et de la santé. Québec: Presses de l'Université du Québec; 2014. 
37. Law M, Cooper B, Strong S, Stewart D, Rigby P, Letts L. The person-environment-occupation model: a transactive approach to occupational performance. Canadian Journal of Occupational Therapy. 1996; 63(1): 9-23.

38. Nussbaum, M.C. Creating Capabilities. The Human Development Approach. Cambridge: The Belknap Press; 2011.

39. Singer, P. Questions d'éthique pratique. Paris: Bayard; 1997.

40. Rawls, J. A Theory of Justice. Cambridge: The Belknap Press; 1971.

41. Aristote. Éthique à Nicomaque. Paris: Vrin; 1959.

42. Drolet MJ. De l'éthique à l'ergothérapie. La philosophie au service de la pratique ergothérapique. $2^{\mathrm{e}}$ éd. Québec : Presses de l'Université du Québec; 2014.

43. Drolet MJ, Hudon A. Theoretical frameworks used to discuss ethical issues in private physiotherapy practice and proposal of a new ethical tool. Medicine, Healthcare and Philosophy. 2015; 18(1): 51-62.

44. Association canadienne des ergothérapeutes (ACE). Profil de la pratique des ergothérapeutes au Canada. Ottawa: CAOT Publications ACE; 2012.

45. Australian Physiotherapapy Council (APC). Australian Standards for Physiotherapy. Safe and Effective Physiotherapy; 2006.

46. CanMEDS. Le Cadre des compétences CanMEDS pour les médecins; 2014

47. Council for Accreditation of Canadian University Programs in Audiology and SpeechLanguage Pathology (CAPUC-ASLP). Profil proposé des compétences relatives à la pratique des orthophonistes au Canada; 2011.

48. Council of Canadian Physiotherapy University Programs (CCPUP). Entry-to-Practice Physiotherapy Curriculum: Content Guidelines for Canadian University Programs; 2009.

49. Groupe consultatif national en physiothérapie (GCNP). Profil des compétences essentielles des physiothérapeutes au Canada; 2009.

50. Drolet MJ, Hudon A. Les professionnels de la santé ont-ils un devoir d'advocacy? Ethica Revue interdisciplinaire de recherche en éthique. 2014; 18(2): 33-63.

51. Drolet MJ. Sur le devoir des professionnels de la santé de défendre les droits des patients. La Quinzaine est Ouest. 2014; 124: 1-12.

52. Drolet MJ, Lalancette M, Caty MĖ. L'ABC de l'argumentation pour les professionnels de la santé : la rhétorique au service de la pratique professionnelle. Québec: Presses de l'Université du Québec; 2015.

53. Leprohon J, Lessard LM, Lévesque-Barbès $H$, Bellavance M. Mosaïque des compétences cliniques de l'infirmière. Compétences initiales. 2e éd. Westmount: Ordre des infirmières et infirmiers du Québec; 2009.

54. United Kingdom Central Council for Nursing, Midwifery and Health Visitors (UKCC). Exercising Accountability. London: UKCC. 1989; 12-13.

55. Drolet MJ, Hudon A (soumis). Le Cadre éthique quadripartite (CÉQ) : un outil pour soutenir le devoir d'advocacy des professionnels de la santé. Ethica - Revue interdisciplinaire de recherche en éthique.

56. Dhillon SK, Wilkins S, Law MC, Stewart DA, Tremblay M. Advocacy in occupational therapy: Exploring clinicians' reasons and experiences of advocacy. Canadian Journal of Occupational Therapy, 2010; 77(4): 241-248.

57. Schwartz Y. L'activité en dialogues - Entretiens sur l'activité humaine (II) suivi de Manifeste pour un ergo-engagement. Toulouse: Octares Editions; 2009. 


\section{Annexe}

\section{Schéma d'entrevue}

N.B. Les questions étaient sujettes à changement par les réponses qui étaient apportées par les participantes et l'adaptation de la chercheuse étudiante en fonction de celles-ci.

\section{A) Exemple (s) de discontinuité dans les trajectoires de soins des individus}

1. J'aimerais que la discussion débute par le partage d'une situation importante que vous avez vécue ou dont vous avez été témoin dans votre pratique clinique concernant une discontinuité dans la trajectoire de soins d'un usager.

2. Croyez-vous que ce genre de situation pourrait survenir dans divers milieux de soins? Si oui, pourquoi?

3. Pouvez-vous décrire les sentiments que vous avez ressentis lorsque la situation que vous avez décrite est survenue?

4. Quels sont les enjeux éthiques (situation de conflit de valeurs ou situations mettant en péril l'actualisation d'une valeur) que soulèvent les situations de discontinuité dans les trajectoires de soins?

B) Les discontinuités dans les trajectoires de soins et services

5. À votre avis, y a-t-il des clientèles plus susceptibles de rencontrer des discontinuités dans leurs trajectoires de soins? Si oui, lesquelles?

6. Y a-t-il des milieux de soins ou des trajectoires de soins parmi lesquels les discontinuités de services sont plus susceptibles de survenir?

7. Croyez-vous que les situations de discontinuité dans les trajectoires de soins peuvent avoir des conséquences positives ou négatives majeures sur les personnes qui les vivent?

8. Si oui, quelles seraient les principales conséquences des situations de discontinuités dans les trajectoires de soins sur les personnes qui ont les ont vécues?

9. Avez-vous déjà été en mesure d'identifier des répercussions sur le plan du rendement, de la participation ou du rendement occupationnel d'une personne ayant vécu une situation de rupture dans sa trajectoire de soins? Pouvez-vous les décrire?

\section{C) Les personnes présentant une problématique complexe de santé.}

10. Y a-t-il des éléments qui facilitent la réponse adéquate à la diversité des besoins d'une même personne?

11. Y a-t-il des éléments qui nuisent à la réponse adéquate à la diversité des besoins d'une même personne?

\section{D) Organisation des services de santé}

12. En tant qu'ergothérapeute, vous êtes-vous déjà restreinte de répondre à tous les besoins occupationnels que vous aviez identifiés chez un client en raison de l'organisation des services de santé ou de votre milieu clinique?

13. Si oui, quelle est la raison précise pour laquelle vous l'avez fait?

14. Advenant le cas où vous auriez eu l'opportunité de répondre à tous ces besoins occupationnels, vous seriez-vous senties outillées pour y répondre? Si non, pourquoi?

\section{E) Pratique de l'ergothérapie}

15. Considérez-vous que votre pratique de l'ergothérapie est holistique? Pourquoi?

16. Y a-t-il des éléments qui facilitent une pratique holistique de l'ergothérapie?

17. Y a-t-il des éléments qui nuisent à une pratique holistique de l'ergothérapie?

18. L'ergothérapeute est-il un professionnel qui a un rôle à jouer dans la diminution des situations de discontinuités dans les trajectoires de soins? 
19. Si oui, que pourrait-il être mis en place, par un ergothérapeute, pour réduire le nombre de discontinuités dans les services et vécues dans les milieux de soins?

20. Est-ce réaliste dans un court délai? Dans un plus long délai?

21. Y a-t-il autre chose que vous aimeriez ajouter? 